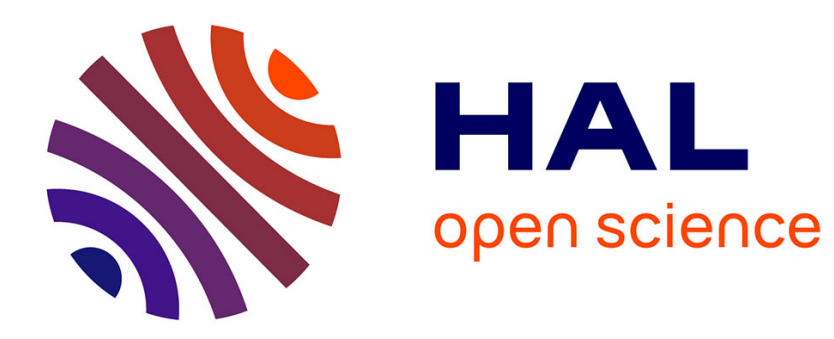

\title{
Stochastic Valuation of Revenue-Collecting Tokens in Cryptoeconomic Organizations \\ Cyprien Grau
}

\section{To cite this version:}

Cyprien Grau. Stochastic Valuation of Revenue-Collecting Tokens in Cryptoeconomic Organizations. 2020. hal-02894497

\author{
HAL Id: hal-02894497 \\ https://hal.science/hal-02894497
}

Preprint submitted on 9 Jul 2020

HAL is a multi-disciplinary open access archive for the deposit and dissemination of scientific research documents, whether they are published or not. The documents may come from teaching and research institutions in France or abroad, or from public or private research centers.
L'archive ouverte pluridisciplinaire HAL, est destinée au dépôt et à la diffusion de documents scientifiques de niveau recherche, publiés ou non, émanant des établissements d'enseignement et de recherche français ou étrangers, des laboratoires publics ou privés. 


\title{
Stochastic Valuation of Revenue-Collecting Tokens in Cryptoeconomic Organizations
}

\author{
Cyprien Grau \\ Head of cryptoeconomics \\ Beem \\ cyprien [@] watchbeem.com
}

June 2020

\begin{abstract}
Cryptoeconomic systems are an emerging type of complex systems that are viewed as a way to steer economic systems and organizations through agents coordination and incentives. However, very little is known about the inherent economic flows of such systems. In this paper, in order to provide intuition on the incentive structures, we draw a first sketch of financial streams in cryptoeconomic organizations. We then present a formal stochastic model of revenue-collecting tokens valuation. With the aim to account for the stochastic nature of this revenue, the financial input of such an organization is assumed to follow a geometric Brownian motion.
\end{abstract}

\section{Introduction}

Cryptoeconomic [1] organizations are social endeavors composed of individuals gathered for a common purpose [2], using a cryptoeconomic infrastructure to automate part of the administration of its policies. As any type of organization, cryptoeconomic organizations are fundamentally value-allocating systems. By allocating part of the value processed to specific tokens, we can define a new class of securities, the revenue-collecting tokens. Here we first present revenuegenerating tokens in the context of financial constraints in cryptoeconomic organizations. We then explicitly model the valuation of revenue-generating tokens and consider the impact on valuation of stochastic financial inputs.

\section{Financial streams in cryptoeconomic organi- zations}

In this section, we first present a simple graphical input-output model of the financial flows in a cryptoeconomic organization to highlight important con- 
straints of these flows. We then introduce more formally revenue-generating tokens, their definition and how they relate to these financial flows and constraints.

Cryptoeconomic organizations process financial streams continuously. By adopting a top-down view of the financial processes within organizations, we can lay down a simple framework presenting cryptoeconomic organizations as financial processing dynamical systems. A cryptoeconomic organization constitutes a framework where its stakeholders give or receive continuous cash flows, forming the financial input and output of these organizations.

The input $I$ is defined as the sum of the operating incomes $C$, investing revenues $R$ and financing proceeds $F$ :

$$
I(t)=C(t)+R(t)+F(t) .
$$

When allocating these inbound streams of cash, cryptoeconomic organizations aim at redistributing as efficiently as possible this cascading financial flow to their stakeholders.

The output $O$ is then defined as the sum of operating expenses $E$, investments $V$ and financing payouts $P$ :

$$
O(t)=E(t)+V(t)+P(t)
$$

To implement time-scale adjustments necessary to a functioning organization (gathering capital for an expected investment for example), a stock and flow model is used. In this model, stocks are formed by designated outputs from the organization and managed by smart contracts, which are self-executing computer programs that ensure the respect of an agreement between two or more parties without the need for a third-party [3]. Here smart contracts act as protocols between the organization and its stakeholders for automatically accruing or distributing financial resources based on a predetermined set of parameters and objectives. In order to mitigate expected future financial outflows, pools of financial resources can then be implemented, becoming provision pools working as stocks. As cryptoeconomic organizations can compute their financial state and distribute financial streams to their stakeholders in near-real time, by extending the basic accounting identity of cash flows [4], we propose that in continuous time, the total financial output $O$ of a cryptoeconomic organization is equal to its total financial input $I$, making this financial input the main limiting financial factor. The financial input is also the most naturally observable economic metric of a cryptoeconomic organization. The goal of these organizations is to optimize the distribution of these financial input and output in order to target an equilibrium that fits all of its stakeholders' preferences and from which resilience emerges over time.

By using a token system, cryptoeconomic organizations can allocate a percentage of their financial input to designated tokens. These revenue-collecting tokens can thus act as a new financing instrument: organizations can sell them to investors, who become token holders and automatically receive a stream of 


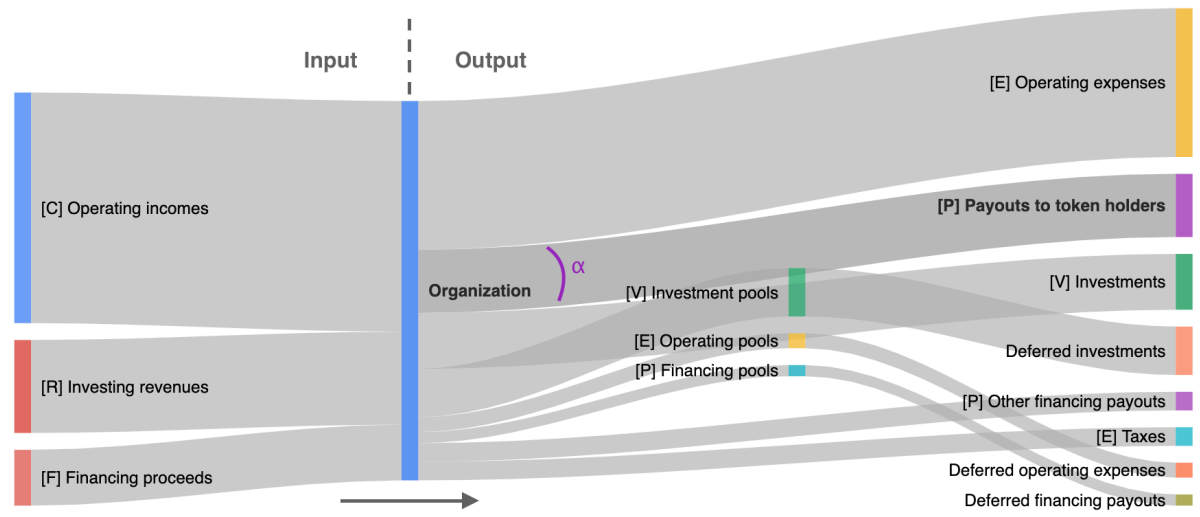

Figure 1: Graphic example of the continuous financial streams of a cryptoeconomic organization

revenue directly correlated with the financial input of the organizations. But these revenue-collecting tokens can also be considered as retributions or rewards for any type of work accomplished within an organization. This includes building and securing the network, in which case the organization can award revenue-collecting tokens to the agents maintaining and improving the system, based on parameters representing the value created over time by these agents within the organization. These parameters are predetermined and executed by smart contracts, but can be updated to follow the needs of the organization, according to the governance protocol upheld by each organization. By defining a certain weight to each stakeholder's claim on a percentage of the financial input (including the financing token holders'), organizations can structure their own cascading revenue flow in order to optimize for their stakeholders' preference.

Because cryptoeconomic organizations can be structured with this type of financial top-down strategy, they can mint revenue-collecting tokens designed to make their holders receive a percentage of the value processed by these organizations (see $\alpha$ in Figure 1), then give these tokens in exchange for some value produced for the organization, or directly sell them to investors for financing purposes. As the cost of financing is directly applied to their top-line, cryptoeconomic organizations are not anymore optimizing for a profit that could have been claimed by shareholders in the context of traditional corporations. By integrating token holders as part of the stakeholders within the cascading revenue flow from scratch, such organizations are able to be profit-less without impeding their financing abilities, making the accounting notion of net profit lose its significance. Of course, this type of economic system makes profit-based taxation policies difficult to implement. To try and give a highly simplified view of the tax issue, for now we suggest to include it within the cascading revenue flow as a revenue-collecting stakeholder (see example of Taxes in Figure 1), which can or cannot be present in the case of Decentralized Autonomous Organizations (DAOs). 


\section{Present value of revenue-collecting tokens}

As seen in Figure 1, financial payouts can be made continuously to the token holders of the organization. To compute the value of the financial right attached to each of these revenue-collecting tokens, we consider a token which grants its holder the right to receive a specific percentage of a continuous financial stream. Below we will denote by $I_{t}$ the total financial input of the minting organization on continuous time $t$, but this process holds for any component of $I$ as defined above. At a given time $t$, token holders are rewarded with a percentage $\alpha$ of the financial input $I_{t}$ of the organization, and with $N_{t}$ being the supply of tokens, the token income stream $S_{t}$ is then:

$$
S_{t}=\frac{\alpha I_{t}}{N_{t}} .
$$

We can then compute the present value $P V_{T}$ of this continuous income stream $S_{t}$ over a holding period of $T$ years, with interest compounded continuously at an annual rate $r$. We start by dividing the interval $[0, T]$ into $n$ subintervals, each of length $\Delta t=T / n$. The endpoints of each subinterval, during which we consider that a single financial transfer is made, are such that $0=t_{0}<t_{1}<\ldots<t_{n}=T$. The present value of the total continuous income stream $S_{t}$ can then be approximated by a Riemann sum of discounted cash flows:

$$
P V_{T} \approx S_{t_{1}} \mathrm{e}^{-r t_{1}} \Delta t+S_{t_{2}} \mathrm{e}^{-r t_{2}} \Delta t+\ldots+S_{t_{n}} \mathrm{e}^{-r t_{n}} \Delta t=\sum_{i=1}^{n} S_{t_{i}} \mathrm{e}^{-r t_{i}} \Delta t .
$$

When $\Delta t \rightarrow 0$, we have $n \rightarrow \infty$ and get:

$$
P V_{T}=\int_{0}^{T} S_{t} \mathrm{e}^{-r t} \mathrm{~d} t,
$$

and by replacing $S_{t}$, we obtain the following present value equation:

$$
P V_{T}=\int_{0}^{T} \frac{\alpha I_{t}}{N_{t}} \mathrm{e}^{-r t} \mathrm{~d} t .
$$

As we might expect, as financial input increases and as holding time $T$ expands, the valuation of a single token goes up, but this valuation decreases with an increase of the total number of tokens minted as well as with higher interest rates.

\section{A stochastic approach to financial input be- havior}

Following the abundant literature on financial derivatives [5], we consider that the financial input $I$ behaves as a stochastic process following a geometric Brow- 
nian motion, with the following characteristics:

- input is non-negative,

- input is continuous in time and value,

- input follows a Markov process (random walk where only the current input level is relevant to predict future input levels),

- past input information is already incorporated in the current input level: the next input movement is conditionally independent of past input movements,

- input movements are normally distributed, and

- input levels are log-normally distributed.

The following formula shows the proportional movement of $I$ :

$$
\frac{\Delta I}{I}=\mu \Delta t+\sigma \epsilon \sqrt{\Delta t} .
$$

The variable $\mu$ is the expected annual percentage of variation of the input, making the first term $\mu \Delta t$ the expected proportional movement, or drift, that the financial input will make during $\Delta t$. The second term follows a random process with $\sigma$ as the expected annual volatility of the input and $\epsilon \sqrt{\Delta t}$ as the random volatility where $\epsilon$ is a random number following a standard normal distribution.

As mentioned above, by using a geometric Brownian motion, we assume that input levels are log-normally distributed. This distribution has a mean of the first term and a standard deviation of the second term, but to adjust for convexity, a correction term $\left(-\sigma^{2} / 2\right)$ is added to the mean. Here we follow Peters and Adamou (2018) [6] and consider $I_{t}$ as the input at time $t$ and $I_{0}$ as the input at time 0 , giving us the following time-dependent log-normal distribution:

$$
\ln \frac{I_{t}}{I_{0}} \sim \mathcal{N}\left[\left(\mu-\frac{\sigma^{2}}{2}\right) t, \sigma \epsilon \sqrt{t}\right] .
$$

Using this log-normal distribution, we can now model the input at time $t$ based on the input at time 0 by

$$
\ln \frac{I_{t}}{I_{0}}=\left(\mu-\frac{\sigma^{2}}{2}\right) t+\sigma \epsilon \sqrt{t}
$$

and rearranging the equation, we get the classic geometric Brownian motion equation for financial input $I_{t}$ :

$$
I_{t}=I_{0} \mathrm{e}^{\left(\mu-\frac{\sigma^{2}}{2}\right) t+\sigma \epsilon \sqrt{t}} .
$$

We can now replace $I_{t}$ in the present value equation found in section 3 , giving us: 


$$
P V_{T}=\int_{0}^{T} \frac{\alpha I_{0} \mathrm{e}^{\left(\mu-\frac{\sigma^{2}}{2}\right) t+\sigma \epsilon \sqrt{t}}}{N_{t}} \mathrm{e}^{-r t} \mathrm{~d} t .
$$

Let us rearrange it and get the present value of a revenue-collecting token as

$$
P V_{T}=\alpha I_{0} \int_{0}^{T} \frac{1}{N_{t}} \mathrm{e}^{\left(\mu-\frac{\sigma^{2}}{2}-r\right) t} \mathrm{e}^{\sigma \epsilon \sqrt{t}} \mathrm{~d} t .
$$

Here we note that as expected, the valuation of a single financing token increases with the expected annual percentage of variation of the input $\mu$. But because of the correction term $\left(-\sigma^{2} / 2\right)$, as the expected annual volatility $\sigma$ increases, the valuation decreases. What is interesting to note is that the token supply $N_{t}$ varies with time, and in certain cases that value can be endogenous to the system. Solving the endogeneity of $N_{t}(P V)$ is beyond the scope of this paper.

\section{Future work}

This paper is a first iteration in working with continuous financial streams within cryptoeconomic organizations. We started by describing a high-level view of financial streams in this type of organizations, then we defined revenue-collecting tokens before moving to a stochastic model of the valuation of this kind of financing tokens. To further refine this model, we plan on integrating stochastic volatility in the model and testing this against actual data. Another work in progress is the bonding curves [7] parametrization for the different types of stakeholders of these organizations. As we have $N_{t}$, the token supply at time $t$, in the present value equation, an addition to the model should aim at integrating the inverse function of the token supply bonding curve with respect to the past and current token market prices into the equation. In another future project, we wish to explore the impact on taxation policies for cryptoeconomic organizations. Finally, we are considering integrating this analysis of financial streams within a broader approach to modelling cryptoeconomic systems' economic as well as non-economic values flows to stakeholders.

\section{References}

[1] Shermin Voshmgir and Michael Zargham. Foundations of Cryptoeconomic Systems. Working Paper Series / Institute for Cryptoeconomics / Interdisciplinary Research 1. Vienna: Research Institute for Cryptoeconomics, Nov. 2019. URL: https://epub.wu.ac.at/7309/.

[2] Michael Zargham et al. Exploring DAOs as a New Kind of Institution. May 2020. URL: https://medium.com/commonsstack/exploring-daos-as-anew-kind-of-institution-8103e6b156d4. 
[3] Nick Szabo. Formalizing and Securing Relationships on Public Networks. 1997. URL: https://firstmonday.org/article/view/548/469.

[4] J. William Petty and John T. Rose. "Free Cash Flow, the Cash Flow Identity, And the Accounting Statement of Cash Flows". In: Journal of Financial Education 35 (2009), pp. 41-55. ISSN: 00933961, 2332421X. URL: http://www.jstor.org/stable/41948624.

[5] John C. Hull. Options, Futures, and Other Derivatives. Pearson, 2018. ISBN: 9780134472089.

[6] Ole Peters and Alexander Adamou. The sum of log-normal variates in geometric Brownian motion. 2018. arXiv: 1802.02939 [cond-mat.stat-mech].

[7] Michael Zargham, Jamsheed Shorish, and Krzysztof Paruch. From Curved Bonding to Configuration Spaces. Working Paper Series / Institute for Cryptoeconomics / Interdisciplinary Research. Vienna: WU Vienna University of Economics and Business, Dec. 2019. URL: https://epub.wu .ac. at/7385/. 